\title{
Cancer incidence attributable to tuberculosis in 2015: global, regional, and national estimates
}

Chi Yan Leung ${ }^{1,2 \dagger}$, Hsi-Lan Huang ${ }^{1,2^{*}+}$, Md. Mizanur Rahman ${ }^{1}$, Shuhei Nomura ${ }^{1,3}$, Sarah Krull Abe ${ }^{1,4}$, Eiko Saito ${ }^{1,2}$ and Kenji Shibuya ${ }^{1,5}$

\begin{abstract}
Background: Tuberculosis is associated with increased risk of cancer. However, the impact of tuberculosis on global cancer burden is unknown.

Methods: We performed random-effects meta-analyses and meta-regressions of studies reporting the association between tuberculosis and cancer risks by searching PubMed, Web of Science, Embase, Cochrane library, and CINAHL from inception to 1 June 2019. Population attributable fractions (PAFs) of cancer incidence attributable to tuberculosis were calculated using relative risks from our meta-analyses and tuberculosis prevalence data from Global Health Data Exchange by age, sex, and country. The study has been registered with PROSPERO (CRD42016050691).

Results: Fourty nine studies with 52,480 cancer cases met pre-specified inclusion criteria. Tuberculosis was associated with head and neck cancer (RR 2.64[95\% Cl 2.00-3.48]), hepatobiliary cancer (2.43[1.82-3.25]), Hodgkin's lymphoma (2.19[1.62-2.97]), lung cancer (1.69[1.46-1.95]), gastrointestinal cancer (1.62[1.26-2.08]), non-Hodgkin's lymphoma (1.61[1.34-1.94]), pancreatic cancer (1.58[1.28-1.96]), leukaemia (1.55[1.25-1.93]), kidney and bladder cancer (1.54[1.21-1.97]), and ovarian cancer (1.43[1.04-1.97]). We estimated that 2.33\%(1.14-3.81) or 381, 035 $(187145-623,404)$ of global cancer incidences in 2015 were attributable to tuberculosis. The PAFs varied by Socio-demographic Index (SDI)—ranging from 1.28\% (0.57-2.31\%) in the high-SDI countries to 3.51\% (1.84-5.42\%) in the middle-SDI countries. Individually, China and India accounted for $47 \%$ of all tuberculosis-related cancer cases.

Conclusions: Tuberculosis is associated with increased risk of cancer at ten sites. The burden of tuberculosis attributable cancer skewed towards lower resource countries. Research priorities are to better understand regional disparities and underlying mechanism linking tuberculosis and cancer development.
\end{abstract}

Keywords: Tuberculosis, Cancer, Attributable fraction

\footnotetext{
* Correspondence: hsilan0728@m.u-tokyo.ac.jp

${ }^{\dagger}$ Chi Yan Leung and Hsi-Lan Huang contributed equally to this work. 'Department of Global Health Policy, Graduate School of Medicine, The University of Tokyo, 7-3-1 Hongo, Bunkyo-ku, Tokyo 113-0033, Japan ${ }^{2}$ Division of Cancer Statistics Integration, Center for Cancer Control and Information Services, National Cancer Center, Tokyo, Japan

Full list of author information is available at the end of the article
}

(c) The Author(s). 2020 Open Access This article is licensed under a Creative Commons Attribution 4.0 International License, which permits use, sharing, adaptation, distribution and reproduction in any medium or format, as long as you give appropriate credit to the original author(s) and the source, provide a link to the Creative Commons licence, and indicate if changes were made. The images or other third party material in this article are included in the article's Creative Commons licence, unless indicated otherwise in a credit line to the material. If material is not included in the article's Creative Commons licence and your intended use is not permitted by statutory regulation or exceeds the permitted use, you will need to obtain permission directly from the copyright holder. To view a copy of this licence, visit http://creativecommons.org/licenses/by/4.0/. The Creative Commons Public Domain Dedication waiver (http://creativecommons.org/publicdomain/zero/1.0/) applies to the data made available in this article, unless otherwise stated in a credit line to the data. 


\section{Background}

In $2015,17.5$ million new cancer cases were reported worldwide, with 8.7 million cancer-related deaths [1]. Carcinogenic infections are well-established risk factors for cancer, namely Epstein-Barr virus, Helicobacter pylori, hepatitis $\mathrm{B}$ and $\mathrm{C}$ virus, human herpes virus type 8 , and human papillomavirus [2]. In 2012, 2.2 million (15.4\%) of global incident cancers were attributed to infections [2]. Substantial reduction of infection-related cancer burden has been made by prevention and treatment of infectious agents, for instance, hepatitis B virus vaccine and human papillomavirus vaccine [2].

Tuberculosis is the global leading cause of infectious disease mortality and the ninth leading cause of death in 2016 [3]. From 2000 to 2016, tuberculosis deaths fell from 1.7 million to 1.3 million, yet an estimated 10.4 million new tuberculosis cases arose in 2016 [3]. Although a growing body of evidence has revealed the association between tuberculosis and cancer, [4-10] the global cancer burden attributable to tuberculosis has not been quantified, and therefore, the potential impact of tuberculosis elimination on cancer burden remains unclear. Quantification of global cancer burden attributable to tuberculosis can contribute to the global and national discussions on health system investments, especially in countries facing the double burden of tuberculosis infection and cancer. In line with the Sustainable Development Goal (SDG) to end tuberculosis, this study aims to quantify the proportion of global cancer incidence in 2015 that was attributable to tuberculosis, and to explore additional potential benefits of tuberculosis elimination.

\section{Methods}

\section{Overview}

We performed a systematic review and meta-analysis to quantify the association of tuberculosis with the risk of cancers. To ensure that population attributable fractions (PAFs) were calculated using pooled risk estimates from sufficient studies, we defined tuberculosis-related cancers as those including more than five studies to synthesise risk estimates and having association with tuberculosis. Then, age-, sex-, and country-specific PAFs of tuberculosisrelated cancers in 2015 were estimated using corresponding pooled relative risks assessed in our meta-analysis. We calculated the PAFs of cancer attributable to tuberculosis in 195 countries and aggregated into 11 geographical regions and five Socio-demographic Index (SDI) categories. This study adhered to the Preferred Reporting Items for Systematic Reviews and Meta-Analyses (PRISMA) guidelines and the Guideline for Accurate and Transparent Health Estimates Reporting (GATHER) (Additional file 1: PRISMA Checklist) $[11,12]$.

\section{Search strategy and selection criteria}

We searched PubMed, Web of Science, Embase, Cochrane library, and CINAHL from inception to 1 June 2019, with no language restrictions, reporting the association between tuberculosis and risk of cancer at 17 sites (Additional file 2: Table S1-S5). In case of non-English articles, we consulted two native speakers for translations. The search strategy was iterative, in that the bibliographies of all included relevant studies were manually searched for additional articles. Two reviewers (CYL and $\mathrm{HLH}$ ) independently conducted title and abstract screening of potentially eligible articles for inclusion. Disagreement on eligibility was resolved by discussion between the reviewers. We included all articles of original observational studies (cohort and case-control studies) which assessed the risk of cancer incidence at 17 sites in patients with tuberculosis compared to those without, starting at age of 20 years or older, and published in a peer-reviewed journal. To minimize potential publication bias, we excluded studies with a sample size of fewer than 50. We specified that each study must either provide relative risk (RR), odds ratio (OR), or hazard ratio (HR) with 95\% confidence intervals (CIs); or provide sufficient data that would allow the risk estimate to be calculated. We excluded reviews, editorials, letters, and animal studies, along with studies assessing cancer mortality risk in tuberculosis infection. The review protocol was registered in PROSPERO (CRD42016050691).

\section{Data extraction and quality assessment}

A standardised observation form (Additional file 2: Supplementary Notes) was independently completed and crosschecked by two reviewers (CYL and HLH) during data extraction. In cases where duplicated cohorts were reported in multiple studies, we extracted data from the study with the larger sample size or higher study quality with a lower risk of bias based on the Newcastle-Ottawa Scale (NOS) [13]. We assessed the methodological quality and risk of bias (Additional file 2: Supplementary Notes) in the selection, comparability, and outcome of all included studies using NOS by two independent reviewers (CYL and HLH) [13].

\section{Statistical analysis}

We estimated pooled cancer-specific RRs with 95\% CIs by random-effects meta-analysis with inverse-variance weighting. OR was converted to RR, [14] and the HR was presumably equivalent to $R R$. We used the adjusted risk ratio from each study unless otherwise specified. We reran random-effects meta-analysis for lung cancer with never-smokers only (Additional file 2: Supplementary Notes) to eliminate the possible confounding effect of smoking. We assessed heterogeneity using $I^{2}$ statistic, where 25,50 , and $75 \%$ were the cut-off value for low, 
moderate, and high heterogeneity, respectively. To explore the source of heterogeneity, we performed random-effects meta-regression to investigate whether associations varied according to geographical region, mean age, quality assessment by Newcastle-Ottawa Scale, sample size, SDI, study design (cohort or case-control study), adjustment for confounding variables, and World Bank countryincome category. Publication bias and small-study effects were assessed by visual inspection of funnel plots and Egger's regression asymmetry test [15]. To address funnel plot asymmetry, we used the trim and fill method to evaluate the number of missing studies and their influence on the pooled estimates. For sensitivity analyses, randomeffects models were re-run without highly influential studies, on the basis of weight estimates from meta-analysis. In this study, unless $P<0.0001$, exact $p$ values are provided.

\section{Tuberculosis attributable fractions}

PAF is the proportion of cancer incidence that can be attributed to a risk factor in a given population [16]. We calculated the PAFs of tuberculosis-related cancers for each sex and age group (20-24, 25-29, 30-34, 35-39, $40-44,45-49,50-54,55-59,60-64,65-69,70-74,75-$ $79,80-84,85-89$, and 90-94) in 195 countries for a binary exposure using the following equation: [16]

$$
(\mathrm{PAF}=) \frac{p(R R-1)}{1+p(R R-1)}
$$

where $p$ is the age- and sex-specific prevalence of tuberculosis in the given population; and $R R$ is the pooled $R R$ of tuberculosis-related cancers estimated in our meta-analyses. Age-, sex-, and country-specific tuberculosis prevalence estimates were derived from Global Health Data Exchange (GHDx) [17]. The case definition contains tuberculosis in all forms, including active tuberculosis and latent tuberculosis infection [17]. For PAF estimation of lung cancer, we restricted to use pooled RR which was adjusted for smoking status. We integrated the uncertainties of estimated RRs and tuberculosis prevalence to report the $95 \%$ CI for PAFs using the substitution method [18].

We estimated age-, sex-, country-, and cancer sitespecific incident cancer cases attributable to tuberculosis infection by multiplying age-, sex-, country-, and cancer site-specific PAFs by corresponding cancer incident cases. We obtained information on age-, sex-, and country-specific cancer incidence from Global Health Data Exchange (GHDx) [17]. Countries and territories were grouped into 11 geographical regions and five SDI quintiles in 2015 (Additional file 2: Supplementary Notes). For regional-specific and SDI-specific PAFs for each cancer site, we divided the summation of individual national estimates of tuberculosis-related cancer incident cases by the total number of cancer incident cases in the corresponding category. The precise time required for the development of tuberculosis-related cancer is not well established. We assumed a lag-time of 15 years between first exposure and cancer diagnosis, which represents the average lag time for most risk factors and cancers [19]. Based on the assumption of lag-time, we mapped the tuberculosis prevalence in 2000 to cancer incidence in 2015. We used STATA version 14.2 (College Station, TX, USA) to analyse data.

\section{Results}

Among 1505 articles identified, 90 were eligible for fulltext review. Search details and process with reasons for exclusion are presented in Fig. 1 and Additional file 2 Table S6. A total of 47 published articles with 49 unique studies reporting on 52,480 cancer cases met the inclusion criteria, providing relevant data on lung cancer risk (38 studies, 40,062 cancer cases) and extrapulmonary cancer risks (13 studies, 12,418 cancer cases) (Additional file 2: Table S7). Overall, 11 of these studies were cohort studies and 38 were case-control studies. The studies were published between 1982 and 2017, with two-thirds (33/49) published after 2000. Eighteen in studies were conducted in Southeast Asia, East Asia, and Oceania; 14 studies in High-income North America; 11 in Western Europe; three in High-income Asia Pacific; and three in Central Europe, Eastern Europe, and Central Asia (Additional file 2: Fig. S1). Quality assessment suggested that $75 \%$ of articles $(35 / 47)$ were at low risk of bias, whereas $5 \%(2 / 47)$ and $20 \%(10 / 47)$ were at medium or high risk of bias, respectively (Additional file 2: Table S8-9, and Fig. S2).

The results from meta-analysis are shown in Fig. 2. Tuberculosis was associated with increased risk of cancer at ten sites: head and neck cancer (RR 2.64 [95\% CI 2.00-3.48]), hepatobiliary cancer (2.43 [1.82-3.25]), Hodgkin's lymphoma (2.19 [1.62-2.97]), lung cancer (1.69 [1.46-1.95]), gastrointestinal cancer (1.62 [1.262.08]), non-Hodgkin's lymphoma (1.61 [1.34-1.94]), pancreatic cancer $(1.58[1.28-1.96])$, leukaemia (1.55 [1.25-1.93]), kidney and bladder cancer (1.54 [1.211.97]), and ovarian cancer (1.43 [1.04-1.97]). The pooled RRs of lung cancer for smoking adjustment and for never-smokers were $1.55(1.31-1.83)$ and 1.64 (1.41$1.91)$, respectively. On the other hand, there was no associations of tuberculosis with breast cancer, central nervous system cancer, cervical cancer, multiple myeloma, malignant melanoma of skin, prostate cancer, thyroid cancer, and uterine cancer. We observed high heterogeneity for lung cancer and malignant melanoma of skin $\left(I^{2}=95.9\right.$ and $78.6 \%$, respectively). Forest plots for each 


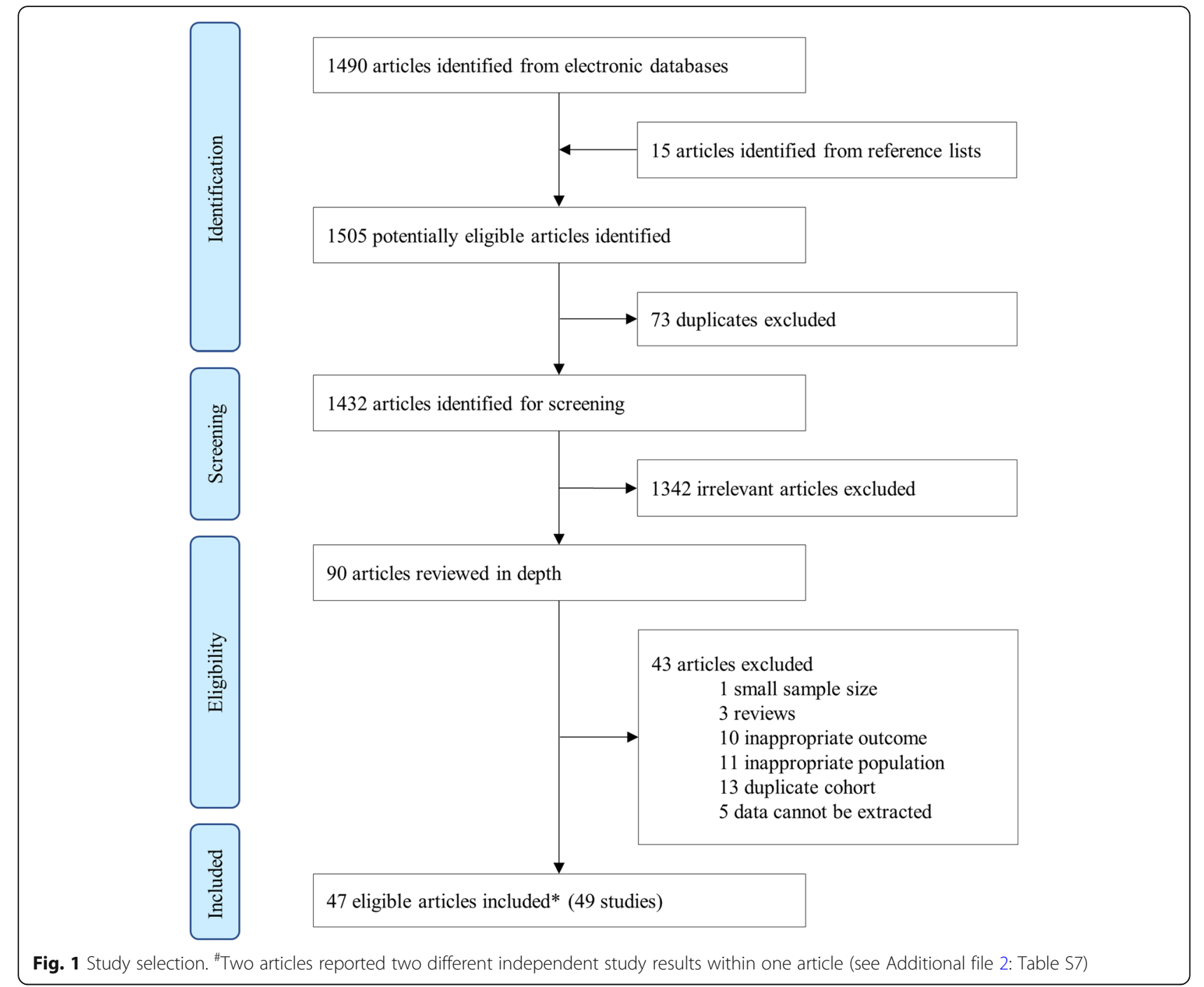

cancer site were presented in appendix (Additional file 2: Fig. S3-9).

Meta-regression analyses (Additional file 2: Table S1013) showed between-group differences by geographical region $(p=0.0305)$ and study design $(p=0.0227)$ for lung cancer, and these two variables explained $37 \%$ of between-study heterogeneity. Associations with tuberculosis were stronger in cohort studies than in casecontrol studies for leukaemia $(p=0.026)$ and nonHodgkin's lymphoma $(p=0.0317)$. Funnel plot asymmetry, which suggests the presence of publication bias and small-study effects, was not evident for lung cancer (Additional file 2: Fig. S10). The trim and fill method in a random-effects model suggested that overall estimates were not greatly modified by publication bias (Additional file 2: Table S14). Sensitivity analyses produced similar results, suggesting that results were robust to exclude highly influential studies (Additional file 2: Table S15).
Among the ten cancer sites identified, we further investigated the PAFs for cancers with pooled RRs obtained from more than five studies. Our results show that an estimated $2.33 \%(1.14-3.81 \%)$ or 381,035 (187145-623,404) of global cancer incidence in 2015 were attributable to tuberculosis infection if the association is causal. By sex, $2.93 \%(1.45-4.75 \%)$ of cancer incidence in 2015 in men and $1.61 \%(0.78-2.67 \%)$ in women were attributable to tuberculosis worldwide (Table 1). PAFs of tuberculosis-related cancers varied by geographical region, SDI, and cancer site. Table 1 shows the regional PAFs, with the highest PAF of 3.99\% (2.16.13) in the Southeast Asia, East Asia, and Oceania, and the lowest PAF of $0.76 \%(0.31-1.45)$ in Australasia. SDIspecific estimates showed that middle-SDI countries had the highest PAF, at $3.51 \%(1.84-5.42)$ of total cancer, while countries with high SDIs had the lowest PAF, at $1.28 \%(0.57-2.31)$ of total cancer (Table 1). Cancer sitespecific estimates varied from $12.59 \%(6.07-21.15)$ for 


\begin{tabular}{|c|c|c|c|c|c|c|}
\hline Outcomes & Studies, No. & Cancer cases & RR $(95 \% \mathrm{CI})$ & Decreased risk & Increase risk & $I^{2}, \%$ \\
\hline Head and neck cancer & 3 & 351 & $2.64(2.00-3.48)$ & & $=$ & 55.6 \\
\hline Hepatobiliary cancer & 3 & 216 & $2.43(1.82-3.25)$ & & $=$ & 16.4 \\
\hline Hodgkin's lymphoma & 6 & 546 & $2.19(1.62-2.97)$ & & $=$ & 0.0 \\
\hline Lung cancer ${ }^{\#}$ & 37 & 40,504 & $1.69(1.46-1.95)$ & & - & 95.9 \\
\hline Gastrointestinal cancer & 3 & 945 & $1.62(1.26-2.08)$ & & $=$ & 0.0 \\
\hline Non-Hodgkin's lymphoma & 8 & 2,390 & $1.61(1.34-1.94)$ & & - & 50.2 \\
\hline Pancreatic cancer & 3 & 152 & $1.58(1.28-1.96)$ & & $\rightarrow-$ & 0.0 \\
\hline Leukaemia & 8 & 2,016 & $1.55(1.25-1.93)$ & & $=-$ & 33.2 \\
\hline Kidney and bladder cancer & 3 & 394 & $1.54(1.21-1.97)$ & & 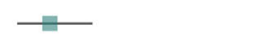 & 0.0 \\
\hline Ovarian cancer & 3 & 79 & $1.43(1.04-1.97)$ & & - & 4.0 \\
\hline Multiple myeloma & 4 & 561 & $1.42(0.90-2.23)$ & & - & 35.0 \\
\hline CNS cancer & 3 & 117 & $1.27(0.98-1.64)$ & & - & 0.0 \\
\hline Prostate cancer & 3 & 639 & $0.98(0.80-1.19)$ & & & 54.3 \\
\hline Malignant melanoma of skin & 4 & 688 & $0.93(0.52-1.65)$ & 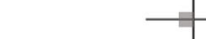 & & 78.6 \\
\hline Breast cancer & 3 & 340 & $0.91(0.78-1.07)$ & -7 & & 0.0 \\
\hline Thyroid cancer & 3 & 42 & $0.88(0.53-1.45)$ & $\rightarrow$ & & 0.9 \\
\hline \multirow[t]{2}{*}{ Uterine cancer } & 3 & 74 & $0.84(0.54-1.31)$ & $\rightarrow$ & - & 23.3 \\
\hline & & & & $\begin{array}{lll} & 0.5 & 1\end{array}$ & $\begin{array}{lllll}1.5 & 2 & 2.5 & 3 & 3.5\end{array}$ & \\
\hline
\end{tabular}

Fig. 2 Summary of pooled relative risks for the association between tuberculosis and cancers. Note: ${ }^{\#}$ Of 37 studies for lung cancer, 23 studies qualified the association between tuberculosis and lung cancer with adjustment for smoking, pooled relative risk (RR) (1.55 [95\% Cl 1.31-1.83], $\left.P^{2}=96.0 \%\right) ; 14$ studies qualified the association between tuberculosis and lung cancer risk among never-smokers, pooled RR $\left(1.64\right.$ [1.41-1.91], $I^{2}=$ 58.8\%). Forest plots for each pooled estimate are shown in Additional file 2 Fig. S3-9. Blue indicates an increase in risk of cancer; grey indicates a null association. No.: number, RR: relative risk, Cl: confidence interval, CNS: central nervous system.

non-Hodgkin's lymphoma to $22.27 \%(10.62-36.44)$ for Hodgkin's lymphoma.

Country-specific PAFs are presented in Fig. 3 and Additional file 2 Table S16. Of the 195 countries we analysed, the PAFs were higher for men than for women in all countries. In men, the PAFs were more than $7.2 \%$ in Morocco, Sudan, and Vietnam; but less than $1.0 \%$ in Australia, Chile, and the United States. In women, the PAFs were more than $4.5 \%$ in North Korea, Sudan, and Vietnam; but less than $0.6 \%$ in Jordan, Malta, and Spain. With respect to the national contribution to tuberculosis-related cancer cases in 2015 (Additional file 2: Table S17), China (153,259 cases [95\% CI 83601-230,298]), India (25,457 [1334138,736]), the United States (19,459 [9532-32,647]), Russia (14,572 [7108-23,676]), and Japan $(12,801$ [6346-21,111]) contributed the most. Two of the top five countries with the highest TB-related new cancer cases were among the three high tuberculosis burden countries listed by the WHO, namely China, and India, accounted for $47 \%$ of tuberculosis-related cancer cases worldwide. When PAFs for lung cancer were adjusted for smoking status, we observed 0.34$3.72 \%$ point difference with comparison to unadjusted PAFs (Additional file 2: Table S18). Since study design is a significant source of heterogeneity for lung cancer and leukaemia, we performed sensitivity analysis to calculate the PAFs using cohort studies exclusively (Additional file 2: Table S19, page 45-47). Compared with estimates in primary analysis, we observed 5.13-15.96 points difference for lung cancer and 3.67-15.31 points difference for leukaemia.

\section{Discussion}

To our knowledge, this study is the first comprehensive assessment to estimate the impact of tuberculosis on global cancer incidence. We performed a systematic review and meta-analysis, synthesising non-overlapping data from 52,480 cancer patients from 49 studies, to quantify the association between tuberculosis and cancer incidence at 17 cancer sites. The study findings show that tuberculosis is associated with increased risk of cancer at ten sites in adults. Our estimates show that $2.93 \%$ $(1.45-4.75 \%)$ of total cancer in men and $1.61 \%(0.78-$ $2.67 \%$ ) in women could be attributed to tuberculosis in 195 countries and territories in 2015.

This study adds important vision to the contribution of infectious agents to cancer risk. Previous study has quantified the global cancer burden attributable to nine infectious agents: Helicobacter pylori, human papillomavirus, hepatitis $\mathrm{B}$ virus, hepatitis $\mathrm{C}$ virus, Epstein-Barr virus, human herpesvirus type 8 , Schistosoma haematobium, Human T-cell lymphotropic virus type 1, and Opisthorchi viverrini [2]. This study is the first estimate of global cancer incidence attributable to tuberculosis infection. The study findings are consistent with and also extend the preceding view on the association between tuberculosis and cancer risk. One previous study estimated the PAF of lung cancer attributable to tuberculosis with $1.1 \%, 2.4$, and $12.7 \%$ in North America, 


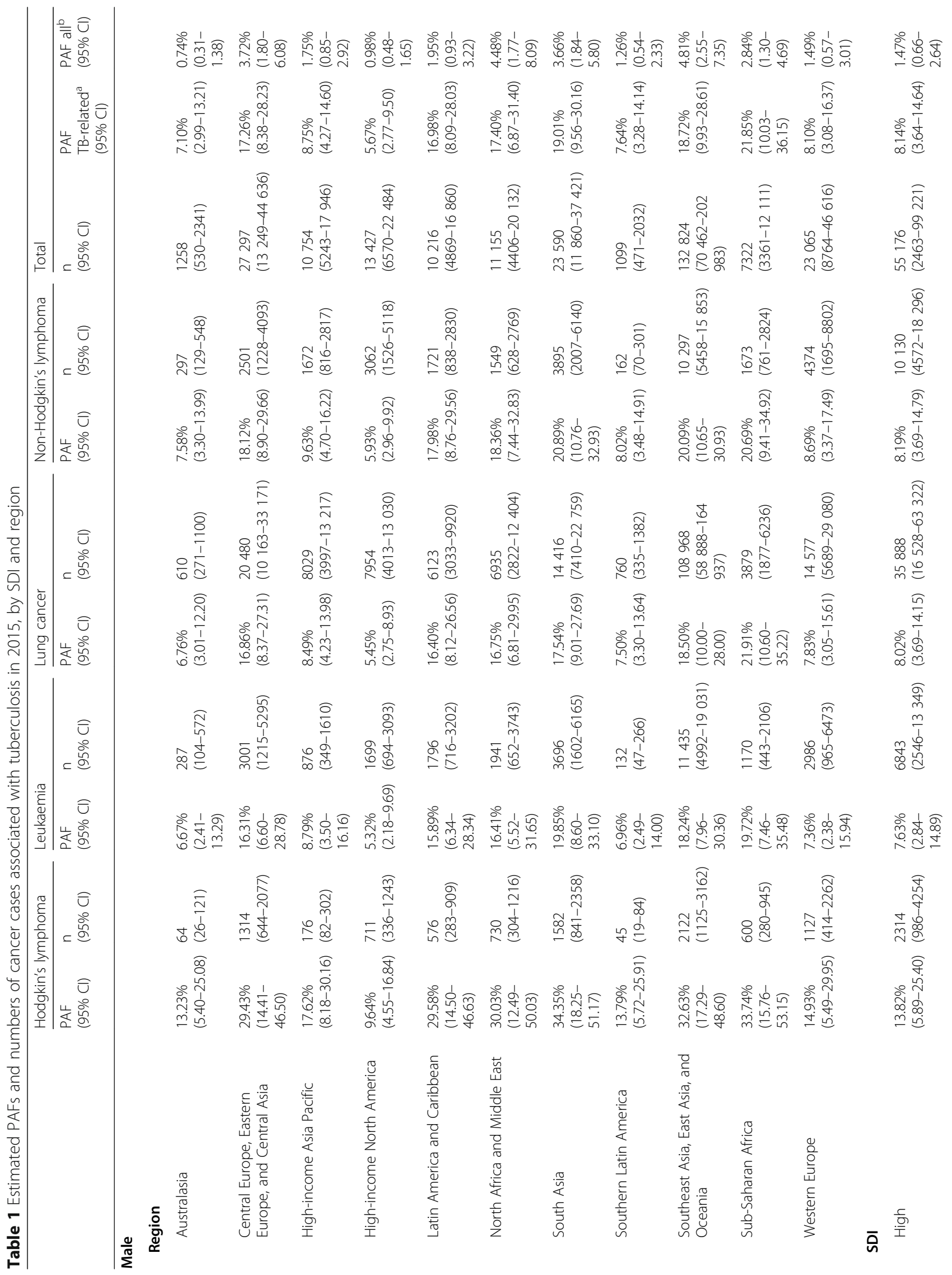




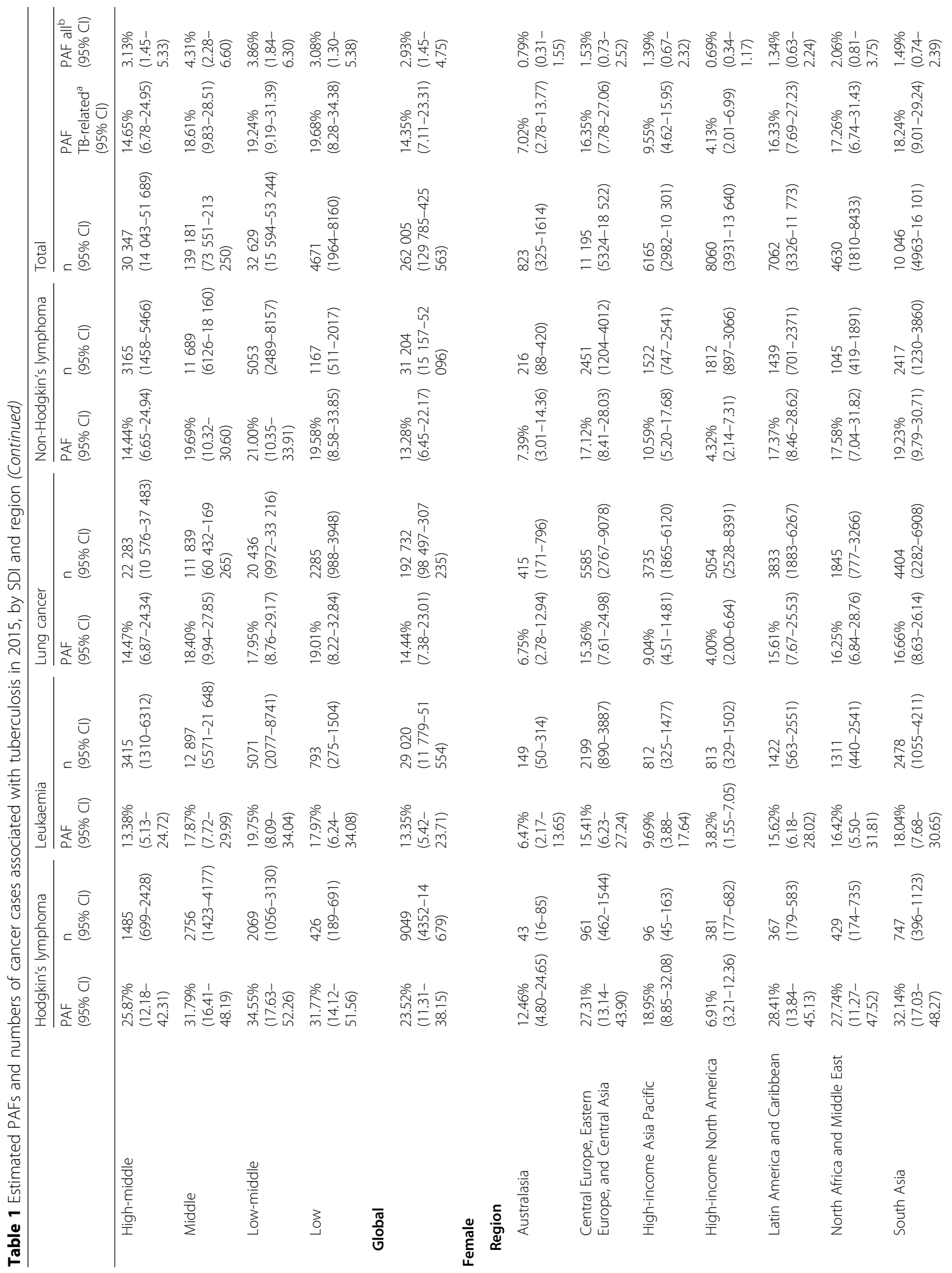




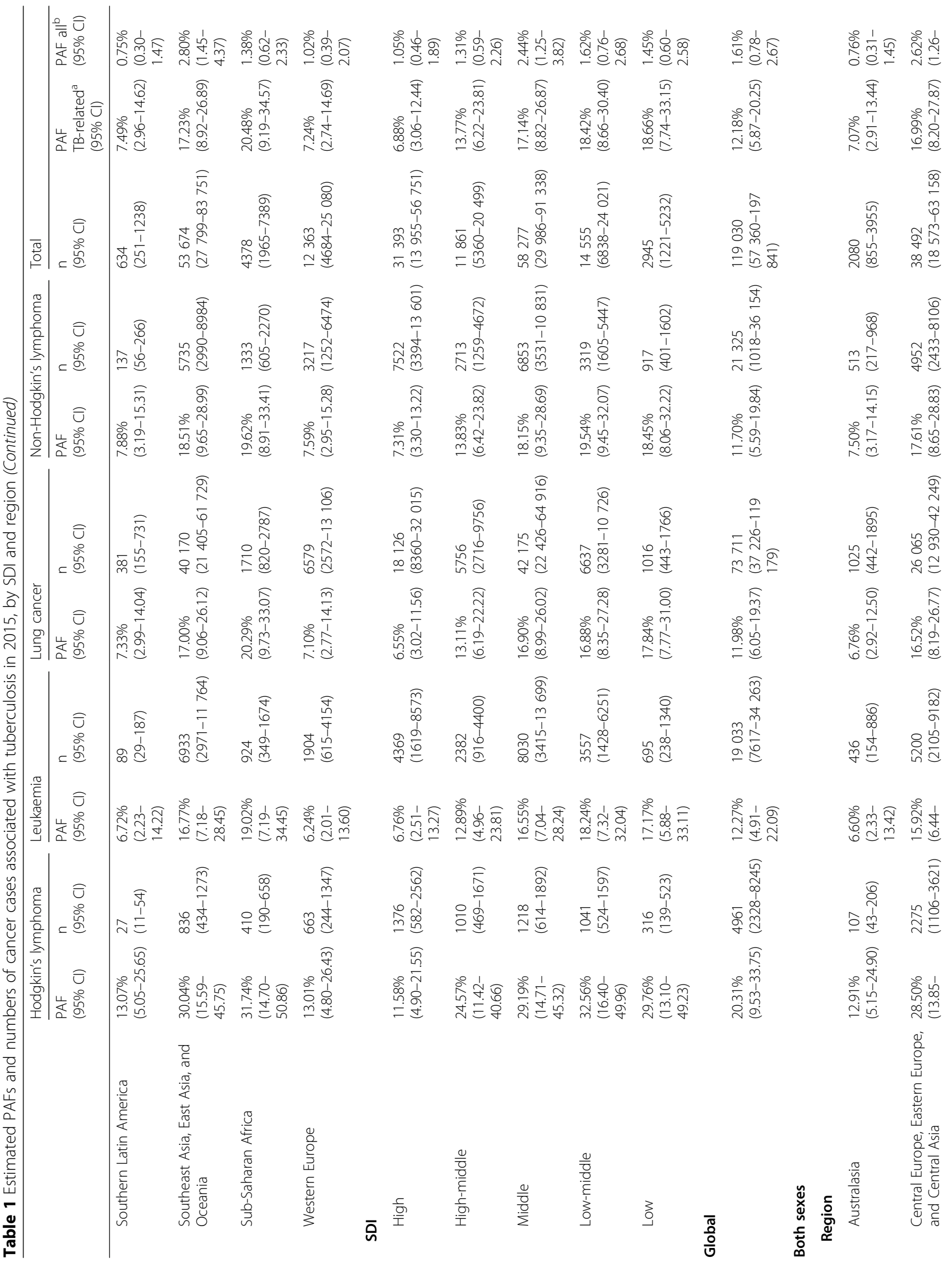




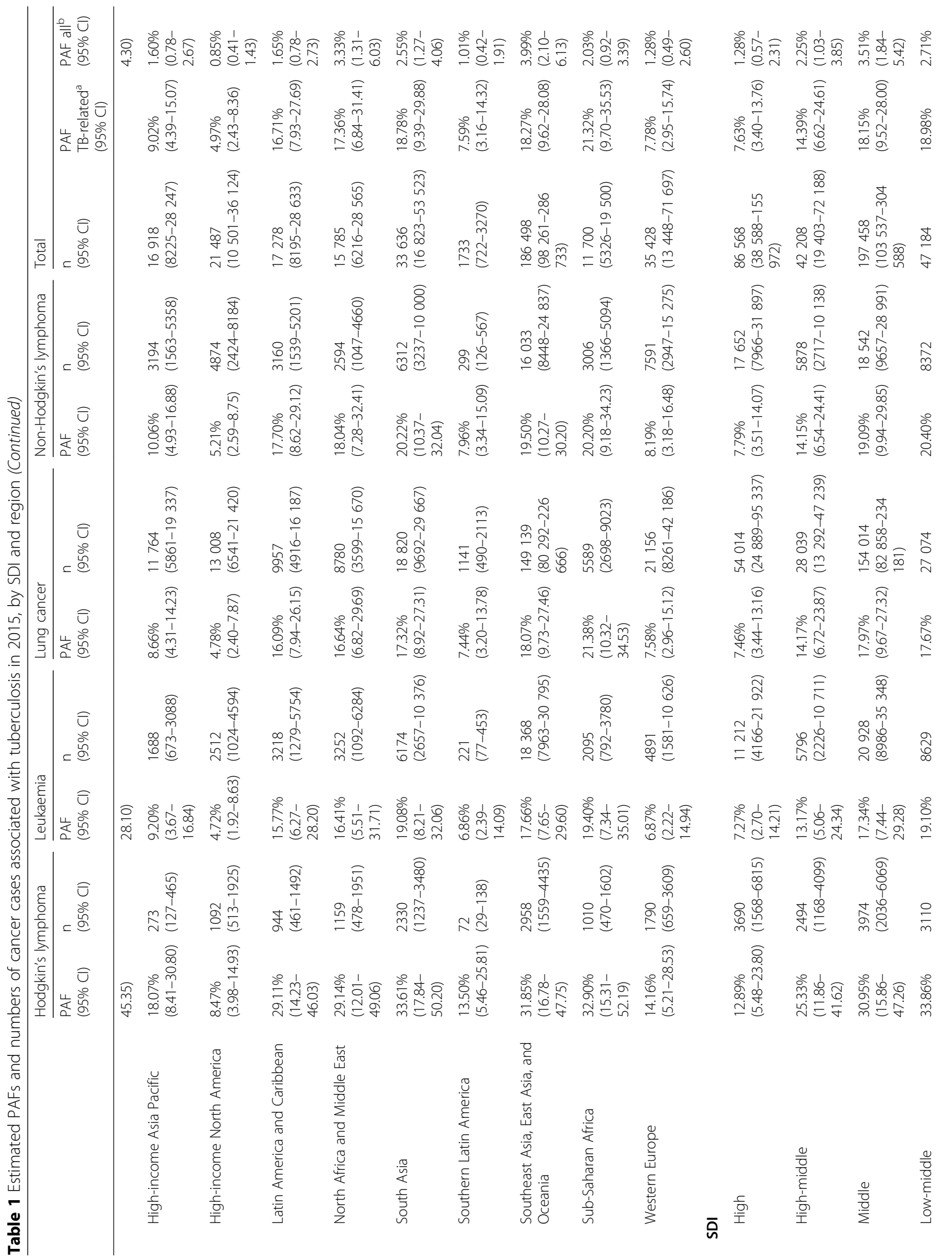




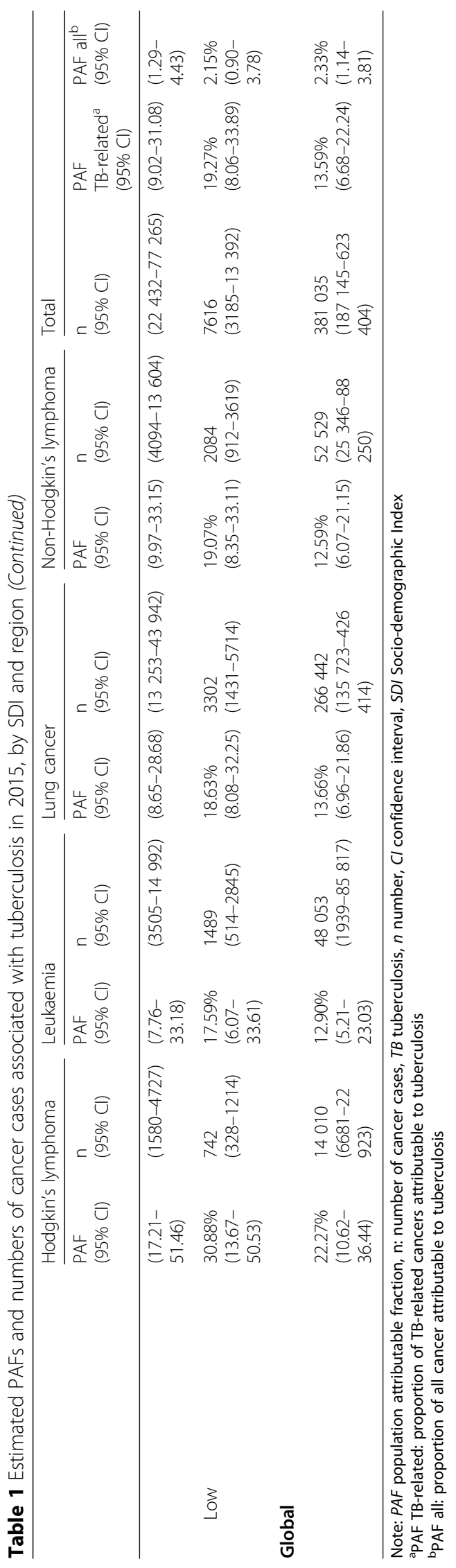




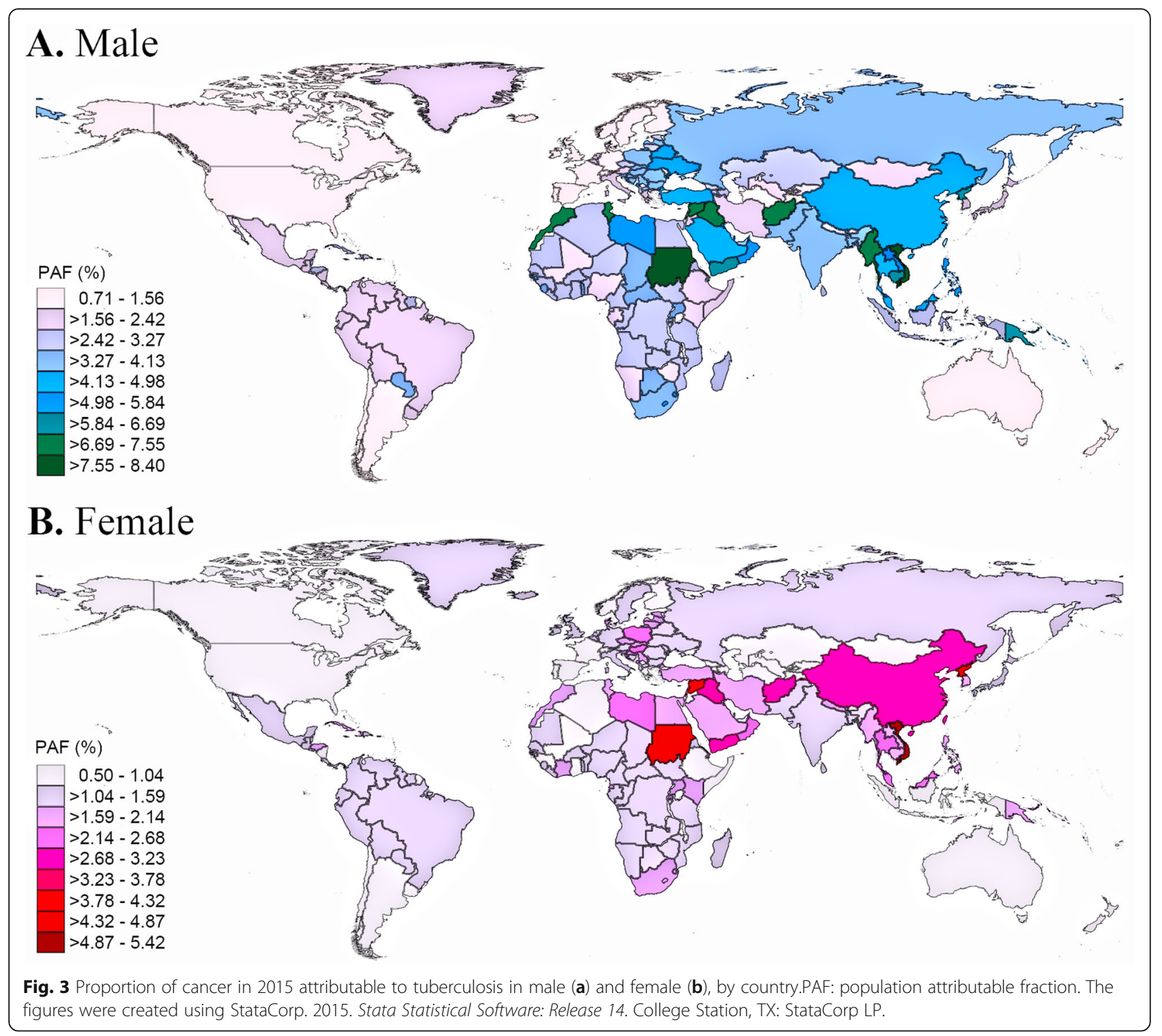

Europe, and China, respectively [20]. This study assessed the PAF for three additional cancer sites and provides sex-, and country-specific estimates with uncertainty intervals and extensive sensitivity analyses compared to previous work. Collectively, our results provide robust estimates derived from a comprehensive search without language restriction and subgroup analyses. Also, we rigorously used the highest quality cancer incidence and tuberculosis prevalence data available.

Chronic inflammation fosters multiple tumourpromoting responses and seeds neoplastic microenvironments [21]. Experimental evidence showed that chronic tuberculosis infection in lung is sufficient to drive carcinogenesis [6]. Genome alteration with DNA damage led by oxidative stress was observed in tuberculosisinfected macrophages 2 months after initial infection. Tuberculosis infection-associated DNA damage, toll-like receptor, and tumour necrosis factor- $\alpha$ activate the nuclear factor $-\kappa B$ pathway and exert an anti-apoptotic effect on DNA-damaged cells [6]. Finally, epiregulin produced by tuberculosis-infected macrophages stimulates the proliferation of surrounding normal epithelial and stromal cells [6]. Similar up-regulated epiregulin expression has also been linked to activation of the Kras signalling pathway in colon cancer [6]. For lung cancer, pathological scarring due to ongoing inflammation might induce carcinogenesis [7]. Also, a relationship between tuberculosis and lung cancer epithelialmesenchymal transition had been demonstrated [8]. Alterations of epithelial cell polarity induce carcinogenesis and are associated with tumour progression [22]. Although, the possibility of reverse causality should be taken into account as compromised immunity in cancer patients may increase the risk of latent tuberculosis 
activation or new tuberculosis infection, [23] reverse causality is unlikely to fully explain the long-term association between tuberculosis and cancer given the increase in cancer risk were observed even five to 20 years after the diagnosis of tuberculosis [24-26]. The underlying causal mechanism remains an active area of research, and more rigorously controlled preclinical studies are needed.

Strategies tailored to country's context for tuberculosis control and elimination might have tremendous potential impact - not merely to reduce the burden of tuberculosis, but also to promote cancer prevention. As China and India account for $47 \%$ of tuberculosis-related cancer cases, the feasibility of these two countries to achieve the WHO target with existing interventions could further impact the global cancer burden [27]. In China, despite the high performance of the nationwide Directly Observed Treatment, Short-Course (DOTS) strategy, epidemic of drug-resistant tuberculosis remains as a major threat to tuberculosis control [28]. Future efforts should be focused on delivering rapid molecular tests for multidrug-resistant tuberculosis and appropriate treatments in peripheral and local health centres to achieve a further reduction in tuberculosis-related cancer incidence. In India, tackling of the key determinants of tuberculosis, such as undernutrition and cigarette smoking, and investment in health systems remain critical priorities to reduce the tuberculosis-related burden [29]. By raising awareness of the importance of the carcinogenic effect of tuberculosis, healthcare policymakers could ultimately lead to the proactive development of measures that positively affect the global cancer burden and therefore contribute to the global public good.

The results should be, however, interpreted with caution. First, meta-analyses of observational studies are susceptible to inherited confounding factors [30]. Smoking is the leading risk factor, [31] and a possible confounder for lung cancer [32, 33]. To overcome this issue, we restricted our PAF calculation of lung cancer using risk estimates adjusted for smoking. The meta-analysis for lung cancer was also re-run with never-smokers to eliminate the effects of smoking. Our risk estimate for never-smokers further suggested that tuberculosis has an independent association with lung cancer. In additional analyses, the adjustment of PAFs for smoking had only small differences in comparison with unadjusted PAFs for lung cancer. Although possible residual confounding cannot be excluded, we have tried to assess the effect of potential confounding using the best available data. Second, the set of studies was heterogeneous, and we could not fully analyse the source of heterogeneity as the individual patient-level data are not available. However, meta-regression showed that geographical region and study design explained $37 \%$ of study heterogeneity of lung cancer. Large prospective cohort studies are needed to further examine the association between tuberculosis and cancer at different sites. Also, future studies performing comprehensive subgroup analyses are warranted. Another limitation of this study is that we did not estimate PAFs separately for active tuberculosis and latent tuberculosis infection because tuberculosis in all form was assessed in 48 of 49 studies included.

\section{Conclusions}

In summary, this study comprehensively explores carcinogenic risk and impact of tuberculosis on global cancer incidence. Our findings reveal that the efforts to achieve the SDG to end tuberculosis would potentially gain additional benefits on reduction of the burden of cancer, particularly in China and India. The present study provides insights into further research to resolve the underlying mechanisms, and to recognise the potential of individual countries to formulate efficient integrated strategies for the preventable burden of tuberculosis and cancers.

\section{Supplementary information}

Supplementary information accompanies this paper at https://doi.org/10. 1186/s12885-020-06891-5.

Additional file 1. PRISMA 2009 Checklist

Additional file 2. Supplementary Notes

\section{Abbreviations}

$\mathrm{Cl}$ : Confidence interval; GHDx: Global health data exchange; HR: Hazard ratio; NOS: Newcastle-Ottawa scale; OR: Odds ratio; PAFs: Population attributable fractions; RR: Relative risk; SDG: Sustainable development goal; SDI: Sociodemographic Index

\section{Acknowledgements}

The views expressed in this paper are solely those of the authors. CYL and $\mathrm{HLH}$ had full access to all the data in the study and takes responsibility for the integrity of the data and the accuracy of the data analysis. The funder of the study had no role in study design, data collection, data analysis, data interpretation, or writing of the manuscript.

\section{Author's contributions}

$\mathrm{CYL}, \mathrm{HLH}$, and $\mathrm{KS}$ conceived and designed the study and protocol registration. CYL and HLH contributed equally to this work. MMR and SKA refined the protocol registration. $\mathrm{CYL}$ and $\mathrm{HLH}$ performed the literature search, data extraction, quality assessment, statistical analysis, and interpretation of data through consultation with MMR. CYL and HLH wrote the manuscript. KS, SN, MMR, SKA, and ES revised the manuscript critically for important intellectual content. All authors approved the final version before submission.

\section{Funding}

This work was partly funded by a research grant from the Ministry of Education, Culture, Sports, Science and Technology, Japan (16H02643). The funder of the study had no role in study design, data collection, data analysis, data interpretation, or writing of the manuscript.

Availability of data and materials Not applicable. 
Ethics approval and consent to participate

Not applicable.

\section{Consent for publication}

Not applicable.

\section{Competing interests}

All authors declare no competing interests.

\section{Author details}

'Department of Global Health Policy, Graduate School of Medicine, The University of Tokyo, 7-3-1 Hongo, Bunkyo-ku, Tokyo 113-0033, Japan. ${ }^{2}$ Division of Cancer Statistics Integration, Center for Cancer Control and Information Services, National Cancer Center, Tokyo, Japan. ${ }^{3}$ Department of Health Policy and Management, School of Medicine, Keio University, Tokyo, Japan. ${ }^{4}$ Epidemiology and Prevention Group, Research Center for Cancer Prevention and Screening, National Cancer Center, Tokyo, Japan. ${ }^{5}$ University Institute for Population Health, King's College London, London, UK.

Received: 5 November 2019 Accepted: 22 April 2020

Published online: 12 May 2020

\section{References}

1. Fitzmaurice C, Allen C, Barber RM, Barregard L, Bhutta ZA, Brenner $H$, et al. Global, regional, and national cancer incidence, mortality, years of life lost, years lived with disability, and disability-adjusted life-years for 32 cancer groups, 1990 to 2015: a systematic analysis for the global burden of disease study. JAMA Oncol. 2017;3:524-48.

2. Plummer M, de Martel C, Vignat J, Ferlay J, Bray F, Franceschi S. Global burden of cancers attributable to infections in 2012: a synthetic analysis. Lancet Glob Health. 2016;4:e609-16.

3. World Health Organization. Global tuberculosis report 2017. Geneva: World Health Organization; 2017. http://apps.who.int/iris/bitstream/10665/25 9366/1/9789241565516-eng.pdf. Accessed 15 Nov 2017.

4. Vento S, Lanzafame M. Tuberculosis and cancer: a complex and dangerous liaison. Lancet Oncol. 2011;12:520-2.

5. Marais BJ, Loennroth K, Lawn SD, Migliori GB, Mwaba P, Glaziou P, et al. Tuberculosis comorbidity with communicable and non-communicable diseases: integrating health services and control efforts. Lancet Infect Dis. 2013;13:436-48

6. Nalbandian A, Yan BS, Pichugin A, Bronson RT, Kramnik I. Lung carcinogenesis induced by chronic tuberculosis infection: the experimental model and genetic control. Oncogene. 2009;28:1928-38.

7. Dheda K, Booth H, Huggett JF, Johnson MA, Zumla A, et al. Lung remodeling in pulmonary tuberculosis. J Infect Dis. 2005;192:1201-9.

8. Gupta PK, Tripathi D, Kulkarni S, Rajan MG. Mycobacterium tuberculosis H37Rv infected THP-1 cells induce epithelial mesenchymal transition (EMT) in lung adenocarcinoma epithelial cell line (A549). Cell Immunol. 2016;300: 33-40.

9. Brenner DR, McLaughlin JR, Hung RJ. Previous lung diseases and lung cancer risk: a systematic review and meta-analysis. PLoS One. 2011;6:e17479.

10. Wong JY, Zhang H, Hsiung CA, Shiraishi K, Yu K, Matsuo K, et al. Tuberculosis infection and lung adenocarcinoma: Mendelian randomization and pathway analysis of genome-wide association study data from neversmoking Asian women. Genomics. 2019;Forthcoming.

11. Moher D, Liberati A, Tetzlaff J, Altman DG, The PRISMA group. Preferred reporting items for systematic reviews and meta-analyses protocols: the PRISMA statement. PLos Med. 2009;6:e1000097.

12. Stevens GA, Alkema L, Black RE, Boerma JT, Collins GS, Ezzati M, et al. Guidelines for accurate and transparent health estimates reporting: the GATHER statement. Lancet. 2016;388:e19-23.

13. Wells G, Shea B, O'Connell D, Peterson J, Welch V, Losos M, et al. The Newcastle-Ottawa scale (NOS) for assessing the quality of non-randomized studies in meta-analyses. 2017. http://www.ohri.ca/programs/clinical_ epidemiology/oxford.asp. Accessed 15 Sept 2018.

14. Zhang J, Kai FY. What's the relative risk? A method of correcting the odds ratio in cohort studies of common outcomes. JAMA. 1998;280:1690-1.

15. Egger M, Davey Smith G, Schneider M, Minder C. Bias in meta-analysis detected by a simple, graphical test. BMJ. 1997;315:629-34.

16. Levin ML. The occurrence of lung cancer in man. Acta-Unio Int Contra Cancrum. 1953;9:531-41.
17. Global Burden of Disease Collaborative Network. Global Burden of Disease Study 2016 (GBD 2016) Results. Seattle, United States: Institute for Health Metrics and Evaluation (IHME); 2017. http://ghdx.healthdata.org/gbd-2016. Accessed 25 May 2018.

18. Daly LE. Confidence limits made easy: interval estimation using a substitution method. Am J Epidemiol. 1998;147:783-90.

19. International Agency for Research on Cancer. Attributable Causes of Cancer in France in the Year 2000. In: IARC working group report, vol. 3. Lyon, France: IARC Scientific Publications; 2007. http://wwwiarcfr/en/publications/ pdfs-online/wrk/wrk3/indexphp 18 May 2018.

20. Sisti J, Boffetta P. What proportion of lung cancer in never-smokers can be attributed to known risk factors? Int J Cancer. 2012;131:265-75.

21. Elinav E, Nowarski R, Thaiss CA, Hu B, Jin C, Flavell RA. Inflammation-induced cancer: crosstalk between tumours, immune cells and microorganisms. Nat Rev Cancer. 2013:13:759-71.

22. Martin-Belmonte F, Perez-Moreno M. Epithelial cell polarity, stem cells and cancer. Nat Rev Cancer. 2011;12:23-38.

23. Cheng MP, Chakra CN, Yansouni CP, Cnossen S, Shrier I, Menzies D, et al. Risk of active tuberculosis in patients with cancer: a systematic review and meta-analysis. Clin Infect Dis. 2017;64:635-44.

24. Brenner AV, Wang Z, Kleinerman RA, Wang L, Zhang S, Metayer C, et al. Previous pulmonary diseases and risk of lung cancer in Gansu Province, China. Int J Epidemiol. 2001;30:118-24.

25. Simonsen DF, Farkas DK, Søgaard M, Horsburgh CR, Sørensen HT, Thomsen RW. Tuberculosis and risk of cancer: a Danish nationwide cohort study. Int J Tuberc Lung Dis. 2014;18:1211-9.

26. Zheng W, Blot WJ, Liao ML, Wang ZX, Levin LI, Zhao JJ, et al. Lung cancer and prior tuberculosis infection in Shanghai. Br J Cancer. 1987;56:501-4.

27. Houben RM, Menzies NA, Sumner T, Huynh GH, Arinaminpathy N, Goldhaber-Fiebert JD, et al. Feasibility of achieving the 2025 WHO global tuberculosis targets in South Africa, China, and India: a combined analysis of 11 mathematical models. Lancet Glob Health. 2016;4:e806-15.

28. Zhao Y, Xu S, Wang L, Chin DP, Wang S, Jiang G, et al. National survey of drug-resistant tuberculosis in China. N Engl J Med. 2012;366:2161-70.

29. Pai M, Correa N, Mistry N, Jha P. Reducing global tuberculosis deaths-time for India to step up. Lancet. 2017;389:1174-6.

30. Egger M, Schneider M, Smith GD. Spurious precision? Meta-analysis of observational studies. BMJ. 1998;316:140-4.

31. Torre LA, Bray F, Siegel RL, Ferlay J, Lortet-Tieulent J, Jemal A. Global cancer statistics, 2012. CA Cancer J Clin. 2015;65:87-108.

32. Collaborators GT. The global burden of tuberculosis: results from the global burden of disease study 2015. Lancet Infect Dis. 2018;18:261-84.

33. Freedman ND, Leitzmann MF, Hollenbeck AR, Schatzkin A, Abnet CC. Cigarette smoking and subsequent risk of lung cancer in men and women: analysis of a prospective cohort study. Lancet Oncol. 2008:9:649-56.

\section{Publisher's Note}

Springer Nature remains neutral with regard to jurisdictional claims in published maps and institutional affiliations.
Ready to submit your research? Choose BMC and benefit from:
- fast, convenient online submission
- thorough peer review by experienced researchers in your field
- rapid publication on acceptance
- support for research data, including large and complex data types
- gold Open Access which fosters wider collaboration and increased citations
- maximum visibility for your research: over $100 \mathrm{M}$ website views per year
At BMC, research is always in progress.
Learn more biomedcentral.com/submissions 